\title{
A colony-level optimisation model provides a potential mechanism for the evolution of novel castes in eusocial ant colonies
}

\author{
Suryadeepto Nag ${ }^{\mathrm{a}}$, Ananda Shikhara Bhat ${ }^{\mathrm{b}, *}$ \\ ${ }^{a}$ Department of Mathematics, Indian Institute of Science Education and Research Pune, \\ Pune-411008, Maharashtra, India \\ ${ }^{b}$ Department of Biology, Indian Institute of Science Education and Research Pune, \\ Pune-411008, Maharashtra, India
}

\begin{abstract}
Ant species often have multiple morphologically distinct 'castes' within a single colony. Given that most of these castes are involved in non-reproductive tasks, and since such individuals thus never reproduce, the question of how ant castes can evolve is a non-trivial one. Over the years, several models have been proposed in order to explain the evolution of castes in ant colonies. Here, we attempt to answer this question using an economics-based approach, developing an optimization model that implements adaptation and selection at the colony level. We argue that due to the nature of ant colonies, selection is shifted to the group level, and, due to this, individual ants are sheltered from negative selection. We show that our framework can explain the evolution of novel castes in ant colonies, and discuss the novelty of our model with regard to previous models that have been proposed. We also show that our model is consistent with several empirical observations of ant colonies.
\end{abstract}

Keywords: ant colonies, group selection, eusociality, evolution, optimization, caste ratio

\footnotetext{
* Corresponding author

Email address: shikharabhat@gmail.com (Ananda Shikhara Bhat)
} 


\section{Introduction}

Darwinian natural selection explains how traits can evolve through time. However, the mechanisms involved behind the evolution of discrete novel traits are relatively less well understood, and often involve proximate mechanisms such as phenotypic or developmental plasticity [1].

Group selection theory, first proposed by Darwin, has increasingly been garnering interest [2], and recent advances have been made both through theoretical approaches [3], and the accumulation of empirical evidence [4] [5]. It has been proposed that since very few members of an ant colony reproduce, the colony as a whole may behave as a 'superorganism', with natural selection being shifted to the colony level[6][7][8]. Evidence for this is also seen through the existence of certain castes that seem to have evolved through ecological specialization, to benefit colony functioning (For example, the door-blocking soldier caste in Cepahlotes[9])

Social hymenopterans often have multiple morphologically and behaviourally distinct 'castes' within the same species. Individuals which have the same genetic code can show greatly differing phenotypes. The presence of such castes is seen in ants, termites[6], bumblebees [10], aphids [11], thrips [12] and the clonal larvae of some parasitic wasps [13] and trematodes [14]. It is thus likely that the presence of castes has convergently evolved multiple times in social animals. However, the mechanism through which novel castes can evolve is unclear, since very few members of the colony reproduce and pass on their genes. Molet et.al. hypothesized that in ants, erratically observed 'intercastes', which are likely developmental recombinations of existing castes, may be the evolutionary precursors which evolve into novel castes [15] [16], but only provided a 'conceptual model' to justify why such intercastes would not be wiped out by evolution. We provide a general mathematical model that justifies the conceptual model that Molet et.al. proposed, and show that our model is also consistent with several empirical observations of the broad patterns seen in ant colonies. 


\section{The Model}

\subsection{The intuition}

The meat of our argument relies on the fact that the costs and benefits of a single non-reproductive individual are likely negligible for survival of the colony. Ant colonies routinely lose workers to both biotic and abiotic factors, and this does not significantly hamper the functioning of the colony. However, if an individual were to somehow acquire a trait such as reproduction, that can be extremely beneficial to survival of the colony even when expressed in only a few members, then the colony benefits. Thus, colonies which produce a few defective worker ants do not pay a severe price, whereas if a colony can produce a few very useful ants, it is likely to greatly increase its own survival, and thus go on to produce more daughter colonies. Thus, if only a relatively small number of individuals in a colony undergo a mutation, the other members of the colony can buffer the entire colony from strong negative selection, acting somewhat analogously to an evolutionary capacitor.

\subsection{Preliminary assumptions}

Assumption 1. We assume that every ant colony is founded by a single 'queen' ant, and attains 'maturity' in finite time

A colony is defined to be mature if it has a well-defined, time-independent size.

Assumption 2. We assume that queens and males produced by these colonies disperse, mate only with ants from other colonies, and go on to produce their own, independent colony.

We define a task as any activity performed by an ant in a colony, that benefits the colony in some way. For example, foraging, defense, and reproduction are all tasks.

Assumption 3. We assume that the ability of an adult ant to perform a task is a function of its morphology, physiology, development, behaviour, or a combination of all of these factors.

Assumption 4. we assume that the types of offspring produced by a queen, and the fractions of offspring that are of each type, are independent heritable traits in queens, and are subject to small mutations.

These assumptions directly imply that natural selection in our model acts at a 'colony-level'. Colonies which produce more queens/males tend to produce more 'daughter colonies', and hence any trait carried by this colony will spread in the population.

Note: The proximate mechanisms evolved in caste determination of Hymenopterans is still being investigated. Some species seem to show a genetic bias, and in others, caste seems to be determined entirely through epigenetic or developmental factors. Regardless of the proximate cause, we assume only 
that the general pattern of caste determination is heritable. For example, if the determination is epigenetic or developmental, then the heritable information is the molecular threshold of stimulation needed in order to determine the caste.

\subsection{Utility Functions}

Consider a mature colony of size $n$, which needs to perform $\eta$ tasks, which are in the ordered set $\mathrm{T}=\left\{\tau_{1}, \ldots, \tau_{\eta}\right\}$. Different tasks have different purposes for the colony. Some tasks like foraging involve work done by ants by expending energy. Others, such as defense of the colony, protect the colony from potential energy losses (due to loss of colony members to predation). Thus, each ant either does some work, or 'saves' some work. We can quantify this (the work done/saved by the $i$ th ant in doing the $j$ th task) as a positive quantity $\omega_{i j}$. We define the Usefulness of the $i$ th ant at performing the $j$ th task as:

$$
u_{i j}:=k_{\tau_{j}} \omega_{i j}
$$

if the ant performs the $j$ th task, and 0 if the ant does not perform the $j$ th task. $\omega$ is a measure of how well an ant performs a given task (and can be empirically quantified in a variety of ways, with the particular metric probably being dependent on the task at hand), and $k_{\tau_{j}}$ is a constant between 0 and 1 which quantifies the importance of the $j$ th task for the functioning of the colony. This is the definition that we use for non-reproductive tasks. If the $j$ th task is reproduction, we instead define the usefulness of the $i$ th ant as:

$u_{i j}:=($ \#offspring produced by focal ant)/(\#offspring produced by queen ant)

, where the 'queen ant' is the ant that acted as the foundress of the mature colony.

We also define the task specific energy consumption of an individual ant as:

$$
e_{i j}=\left(\text { Energy spent by } i \text { th ant in performing } \tau_{j}\right)
$$

We define the competence $c_{i}$ of the $i$ th ant as:

$$
c_{i}:=\sum_{j=1}^{\eta}\left(u_{i j}-e_{i j}\right)
$$

We also define the utility $\mathcal{U}_{j}$ of a task $\tau_{j}$ in the colony as:

$$
\mathcal{U}_{j}:=\sum_{i=1}^{n}\left(u_{i j}-e_{i j}\right)
$$

For large values of $n$, it may be useful to define the fraction of the population performing the task $i$ as $\epsilon_{i}:=\frac{n_{i}}{n}$ where $n_{i}$ is the total number of individuals 
in the colony with non-zero individual utility for the task $\tau_{i}$ (i.e the number of individuals which perform the task $\tau_{i}$ ). We define the fractional marginal utility as:

$$
\nu_{i}\left(\epsilon_{i}, \Delta \epsilon_{i}\right):=\frac{\mathcal{U}_{i}\left(n \cdot\left(\epsilon_{i}+\Delta \epsilon_{i}\right)\right)-\mathcal{U}_{i}\left(n \cdot\left(\epsilon_{i}\right)\right)}{\Delta \epsilon_{i}}
$$

where $\Delta \epsilon_{i}$ is a small change in $\epsilon_{i}$. In the infinitesimal limit, we have:

$$
\mu_{i}:=\lim _{\Delta \epsilon_{i} \rightarrow 0} \nu_{i}\left(\epsilon_{i}, \Delta \epsilon_{i}\right)=\frac{\partial \mathcal{U}_{i}}{\partial \epsilon_{i}}
$$

\subsection{Productivity and consumption functions}

We next define the Gross Productivity function of a colony as:

$$
\mathcal{G}:=\sum_{i=1}^{n} \sum_{j=1}^{\eta}\left(u_{i j}-e_{i j}\right)=\sum_{i=1}^{n} c_{i}=\sum_{j=1}^{\eta} \mathcal{U}_{j}
$$

Thus, the competence of an individual is a measure of how much the presence of that individual contributes to the gross productivity of the colony, and the utility of a trait is a measure of how much the presence of the trait contributes to the gross productivity of the colony.

We define a subsistence function $\mathcal{E}(t)$ for the colony as:

$$
\mathcal{E}(t):=\sum_{i=1}^{n} E_{i}
$$

where $E_{i}$ is the energy that needs to be consumed by the $i$ th ant of the colony in order to survive, when it is not performing any tasks. The value of $E_{i}$ will depend on the morphology of the $i$ th ant, and, in practical terms, can be measured by determining the basal metabolic rate of the $i$ th ant.

Assumption 5. We assume that $\mathcal{E}$ grows linearly with an increase in $n$, when $\epsilon_{i}$ is held constant for all tasks.

This is a reasonable assumption to make, since each individual ant of a certain morphological type on average consumes the same amount of energy, and, if $\epsilon_{i}$ is kept constant for all tasks, then, by consequence, the fraction of ants which have a particular morphology is also kept constant.

We also define a Net Productivity function $\mathcal{P}(t)$ as follows:

$$
\mathcal{P}(t):=\mathcal{G}(t)-\mathcal{E}(t)
$$

Assumption 6. Crucially, we assume that given a colony of size $n$, the percapita net productivity $p(t)=\frac{\mathcal{P}(t)}{n}$ acts as a maximand for selection, i.e natural selection tends to select colonies which have higher per-capita net productivity. Explicitly, if a colony has net productivity $p$ and fitness $w$, we assume that:

$$
\frac{d w}{d p}>0
$$


Alternately given a colony of size $n$ with net productivity $\mathcal{P}$,

$$
\left(\frac{\partial w}{\partial \mathcal{P}}\right)_{n}>0
$$

Note that here, by 'fitness', we refer to the absolute reproductive fitness of a colony, measured by the number of daughter colonies that it produces.

Assumption 7. We assume that for any given task $\tau_{i}$, the utility function $\mathcal{U}_{i}$ follows the law of diminishing marginal utility past a threshold both when varied with $n_{i}$ (keeping $\epsilon_{i}$ constant for all tasks $\tau_{i}$ ), and when varying any given $\epsilon_{i}$ (when $n$ is kept constant), i.e:

$$
\begin{array}{ll}
\left.\frac{\partial^{2} \mathcal{U}_{i}}{\partial n_{i}^{2}}\right|_{\epsilon_{i}}<0 & \forall n_{i}>n_{i_{0}} \in \mathbb{N}, \forall \tau_{i} \in \mathrm{T} \\
\left.\frac{\partial^{2} \mathcal{U}_{i}}{\partial \epsilon_{i}^{2}}\right|_{n}<0 & \forall \epsilon_{i}>\epsilon_{i_{0}} \in[0,1], \forall \tau_{i} \in \mathrm{T}
\end{array}
$$

This implies that $\mathcal{G}$ follows a similar behaviour past a threshold i.e.

$$
\left.\frac{\partial^{2} \mathcal{G}}{\partial n^{2}}\right|_{\epsilon_{i}}<0 \quad \forall n>n_{0} \in \mathbb{N}
$$

Assumption 8. we further assume that the following trends hold:

$$
\begin{aligned}
\lim _{n_{i} \rightarrow \infty} \frac{\partial \mathcal{U}_{i}}{\partial n_{i}} & =0 & \forall \tau_{i} \in \mathrm{T} \\
\lim _{\epsilon_{i} \rightarrow 1} \frac{\partial \mathcal{U}}{\partial \epsilon_{i}} & =0 &
\end{aligned}
$$

It is intuitively easy to see why assumptions 7 and 8 are reasonable. They are justified by the fact that the resources around an ant colony are generally finite. Thus, if, for example, we were looking at the individual usefulness of foragers, we would expect that indefinitely increasing the number of nest members or the number of foragers would not keep increasing the utility of foraging at a constant rate. In fact, as you keep adding foragers, you would expect the resources to become increasingly limited, leading to logistic-like growth of the utility functions.

Since $\mathcal{G}=\sum_{j=1}^{\eta} \mathcal{U}_{j}$, we have:

$$
\lim _{n \rightarrow \infty} \frac{\partial \mathcal{G}}{\partial n}=0
$$




\section{Results}

\subsection{Division of labour}

Consider a mature colony of size $n$. Let the fraction of individuals which carry out a given task $\tau_{i}$ be given by $\epsilon_{i}$. By assumption (6), we look for the condition at which $\nabla \mathcal{P}=0$.

We define the Marginal rate of substitution (MRS) $M_{i j}$ between two tasks as:

$$
M_{i j}=\frac{\mu_{i}}{\mu_{j}}
$$

Where $\mu_{i}$ and $\mu_{j}$ are the fractional marginal utilities of the $i$ th and $j$ th task respectively. Rearranging, we get,

$$
\mu_{i}=M_{i j} \mu_{j}
$$

This is a comparison of the marginal utilities of task $\tau_{i}$ and $\tau_{j}$ and tells us that adding one more ant to perform task $\tau_{i}$ is " $M_{i j}$ times as useful" as adding one ant to perform task $\tau_{j}$ instead, in terms of contributions to the gross productivity of the colony.

By assumption (7), we see that as $\epsilon_{j}$ keeps increasing (keeping $\epsilon_{i}$ fixed), $\mu_{j}$ keeps reducing. Thus, $M_{i j}$ increases, and it is more beneficial to the colony if ants start performing $\tau_{i}$ instead. This is how division of labour evolves. i.e. it is more useful if at any given time, different groups of ants perform different tasks, instead of all ants performing the same task.

It is evident that an equilibrium will be attained only when the marginal utility of both tasks is equal, i.e there can be no further increase in $\mathcal{P}$ by individuals switching from one task to the other. This means that $M_{i j}=1$

Extending this to $\eta$ tasks, we see that the fixed proportions of workers of a colony that perform a given task can be determined by solving:

$$
M_{i j}=1 \forall \tau_{i} \neq \tau_{j} \in \mathrm{T} \Longrightarrow \mu_{1}=\mu_{2}=\ldots \ldots=\mu_{\eta}
$$

, constrained to the condition:

$$
\sum_{i=1}^{\eta} \epsilon_{i} \leq 1
$$

This condition is true because at any given instant of time, an ant can perform atmost 1 task. Note that (3.1) is a constraint on the colony as a whole, and not on individuals. Therefore, an ant which engages in task $\tau_{i}$ at some point of time, can switch over to task $\tau_{j}$ at some later point of time, as long as this switch is also accompanied by some other ants also switching tasks. 


\subsection{Redistribution of tasks helps the colony}

Here we study what happens if a fraction of individuals who perform a particular task die. We show that in our system, a colony redistributes its workers such that this loss is supplemented by a readjustment of workers from other tasks.

Let us assume that some number of individuals which performed the task $\tau_{i}$ died. Before any individuals died, the system was in equilibrium, and equation (3.1) held, i.e $M_{i j}=1 \forall \tau_{j} \neq \tau_{i} \in \mathrm{T}$. Now, if $\delta \epsilon_{i}$ is the fraction of dead workers out of the initial $\epsilon_{i_{0}}$, we see that:

$$
\begin{gathered}
\epsilon_{i_{0}}-\delta \epsilon_{i}<\epsilon_{i_{0}} \\
\left.\Longrightarrow \frac{\partial \mathcal{U}_{i}}{\partial \epsilon_{i}}\right|_{\epsilon_{i_{0}}-\delta \epsilon_{i}}>\left.\frac{\partial \mathcal{U}_{i}}{\partial \epsilon_{i}}\right|_{\epsilon_{i_{0}}} \quad \text { (By assumption (7)) }
\end{gathered}
$$

Thus, we see that $M_{i j}>1 \forall \tau_{j} \neq \tau_{i} \in \mathrm{T}$, and a 'redistribution' of workers such that more workers are allocated to perform $\tau_{i}$ would increase the productivity of the colony. It is easily seen that even after this redistribution, the new net productivity is still less than the productivity of the colony before the deaths occured. Thus, this type of redistribution only serves to 'buffer' the loss of the dead individuals.

\subsection{Main proof}

We define a caste as a morphologically and behaviourally specialised type of ant, which performs a particular, fixed set of functions in the colony. We begin by examining our fitness function $w(t)$, which measures the average number of daughter colonies produced by a given colony at a given point of time. If $w(t)$ is greater than 1, a colony will not go extinct and the genes of its foundress are passed on in the population. We examine the rate of change of fitness,

$$
\varphi:=\frac{d w}{d t}
$$

For the sake of simplicity, we will assume that we start with a mature colony that has only a queen and a single other caste, which we shall call the 'worker' caste. The same arguments proposed here hold regardless of the initial state of the colony, as will easily be seen. Consider such a colony of size $n$ which has a basal fitness of $w_{0}$. Let a mutation occur in the daughter colony produced by this queen. By assumption (4) this mutation could affect either the types of non-reproductive offspring produced, the fraction of each of those types of offspring produced, or both. Let us assume both factors are affected, and ants which possess a previously unseen trait (or combination of traits) are now born in this colony. If this mutation increases the competence of these ants, it automatically increases the fitness of the colony, and this trait will spread in the 
population, and we are done. Let us assume that this mutation decreases the fitness of the colony, by negatively affecting the competence of the ants that are produced. Let the competence of the new ants be $c_{n}(t)$. It is clear that the rate of change of fitness will depend on the fraction of offspring who are affected. Let the number of such offspring produced at time $t$ be $m(t)$.

Now, at every instance in which the colony (or its descendents) breeds, there is some chance that there is another mutation which further modifies this trait. Thus, this trait is now effectively performing a random walk in mutation-space. Let $\theta_{-}$be the time elapsed before this trait becomes beneficial to the colony, and the marginal net productivity becomes positive again. Qualitatively, this is the pount at which ants which express the trait are 'atleast as useful' as a worker. If, in this time, the fitness never dropped to below 1 , the trait is never removed and is successfully fixed in the population. Let us assume that the fitness drops below 1 before the trait becomes beneficial. From the point when fitness first started increasing again, let $\theta_{+}$be the time taken before the fitness grows back to 1 .

Thus, the total time elapsed is $\theta=\theta_{-}+\theta_{+}$, and the total change in fitness $\Delta w$ during this period will be given by:

$$
\Delta w=\int_{0}^{\theta} \varphi d t
$$

Now, this trait can be removed from the population at two time periods:

Case 1: Extinction during $\theta_{-}$:

In the entire period of $\theta_{-}$, the fitness is continually decreasing. Thus, if

$$
\int_{0}^{\theta_{-}} \varphi d t \geq w_{0}
$$

the trait will be removed from the population, since the fitness of the colony is reduced to 0. Biologically, this either means that the trait was so bad that it drastically reduced the fitness of the colony in a short period of time, or that the trait either took too long to become beneficial to the colony, or was not expressed in high enough numbers to become beneficial to the colony.

\section{Case 2: Extinction during $\theta_{+}$:}

Assuming that the population reaches the point where the trait becomes beneficial without going extinct, it can still go extinct if the fitness does not rise back up quickly enough. Thus, if the total number of colonies produced at the end of time $\theta_{-}$is $N_{-}$and the time averaged fitness in subsequent time $\phi$ is given by $\overline{w_{\phi}}$, then the condition for extinction is that:

$$
N_{-} \cdot\left(\overline{w_{\phi}}\right)^{\phi} \rightarrow 0
$$


Let us assume that this, too, doesn't happen, and the population successfully reaches a fitness of 1 . We would like to know how to maximise the chances of reaching this stage.

We now draw on assumption 6 , and assume that examining how productivity changes will allow us to draw inferences about how the fitness changes. We now have exactly two kinds of ants: Those that have the mutation, and those that don't. We can safely assume that all the ants which have the trait are identical to each other, and that, likewise, all ants which do not have the trait are also identical to each other. Thus, our productivity can be split into two terms: Let $p_{m}$ be the per capita productivity of the ants which possess the mutation, and let $p_{0}$ be the per capita productivity of the ants which do not possess the mutation (This is why we assumed a colony with only one kind of worker. If we do not, we will have several different $p_{0}$ terms, but the basic arguements do not change). Now, we have:

$$
\mathcal{P}(t)=m(t) p_{m}(t)+(n-m(t)) p_{0}
$$

Differentiating equation (3.3) with respect to $\mathrm{m}$ gives us:

$$
\frac{d \mathcal{P}}{d m}=p_{m}-p_{0}
$$

and differentiating equation (3.3) with respect to time gives us:

$$
\frac{d \mathcal{P}}{d t}=m \frac{d p_{m}}{d t}+\frac{d m}{d t}\left(p_{m}-p_{0}\right)
$$

We now have the necessary equations, and can examine the optimal conditions at each phase of this process.

Phase 1: $0<t<\theta_{-}$

During this period, the trait is not useful, and $p_{m}$ is less than $p_{0}$. Thus, the quantity $p_{m}-p_{0}$ is negative. A cursory glance at equation (3.4) tells us that at this stage, $\frac{d P}{d m}$ is a decreasing function. Thus, mutations that minimise $m$ tend to maximise $\mathcal{P}$ (and hence fitness, since we are assuming that $n$ is constant). Examining equation (3.5) also tells us that either small positive values, or large negative values of $d m / d t$ will tend to increase $\mathcal{P}$. Thus, up until the trait becomes beneficial, it should only affect a small proportion of the population.

Phase 2: $\theta_{-}<t<\theta_{+}$

During this trait, the trait is useful. Thus, the quantity $p_{m}-p_{0}$ is positive.

Examining equation (3.4), we see that $\frac{d \mathcal{P}}{d m}$ is now positive, and hence, increasing $m$ will increase $\mathcal{P}$ (and hence fitness). Additionally, examining equation (3.5) tells us that we must now try to maximise $d m / d t$. For as long as $\frac{d p_{m}}{d t}$ is 
positive (i.e for as long as the trait becomes more and more useful with time), maximising it is beneficial. Thus, in this phase, populations in which $m$ and $p_{m}$ both increase rapidly will be less likely to go extinct, which is in line with what we expect intuitively.

In conclusion, we have shown that in our model, novel complex traits can evolve in ant colonies if the following conditions are satisfied:

(i) The trait is not initially severely detrimental to the fitness of the colony.

(ii) The trait can,when developed, in principle become useful to the colony even when expressed in small numbers.

(iii) The trait is initially expressed in only a small number of the non-reproductive offspring, until it becomes useful.

(iv) Once the trait becomes beneficial, its presense in the population increases rapidly

The trait may still evolve if (iv) is not satisfied, and biologically, we expect $\frac{d p_{m}}{d t}$ to be small, since mutations are not saltatory. In real life, ants which satisfy these conditions are the 'intercastes', which often have reduced utility, and are produced erratically in small numbers. In fact, both the soldier caste and the ergatoid queen caste exhibit the tell-tale signs of having developed from developmental mosaics of worker and queen castes [15]. Thus, our model justifies the conceptual model proposed by Molet et.al.

Note that our model predicts that $m$ should continually increase only until $p_{m}=p_{0}$ (i.e the per capita productivity of both types of ants is equal)

\section{Discussion}

Our model relies on several assumptions, some more realistic than others. Some of these assumptions may seem too idealistic. At this stage, we would like to point out that most mathematical models, due to the nature of model construction, must tread a fine line between idealism and realism. A model that is too realistic risks losing predictive power by having to incorporate too many parameters, and a model that relies on too many assumptions risks losing accuracy in the real world. Unfortunately, this is a limitation of using mathematics to make predictions about complex, real-world phenomena. We hope that we have managed to tread this fine line, in the sense that while some of our assumptions may not be very realistic, they may help provide a model that is atleast a crude approximation of the real world. Our model is not intented to be right in all its details, it is only intended to be useful and/or insightful. 


\subsection{Comparisons with previous models}

To the best of our knowledge, the only model for how novel (discrete) castes may evolve in ant colonies was provided by Molet et.al.[15][16]. Molet et.al. proposed a conceptual model to explain the evolution of novel castes, and claimed that small mutations in a few ants could be buffered by the rest of the colony. Our model is a mathematical model that starts from very general assumptions, and mathematically shows that the conceptual model that they propose is justified.

Our model also shares some similarities with other optimization models that have been proposed in the past. Oster and Wilson developed an ergonomic theory to determine which castes should be maintained in a population, and which should be eliminated [17][18]. This was accomplished primarily through a linear optimization model, which lead to several predictions. The model that we present is a more general optimization model than that of Oster and Wilson, but some of the predictions that the two models make are the same. The most important prediction that the two models make is the same: The ratio of castes that are present in a colony should be close to the optimum value. Though empirical evidence is relatively scarce, support for optimal caste ratio theory is seen in ants[19][20][6], termites[6] the clonal larvae of polyembryonic wasps[21], and the clonal larvae of trematodes [22][23][24][25].

Hasegawa also developed an optimizatiom model, that relied on a multidimensional hyperspace[19]. However, in Hasegawa's model, the net 'efficiency' (which is equivalent to the net productivity function in our framework) is given by the product of the individual efficiencies. That is to say, if there are $\tau$ tasks in a colony, and if the caste ratio of the colony is $r$, then, in Hasegawa's model, the total colony efficiency is given by $\prod_{i=1}^{\tau} \alpha_{i} t_{i}(r)$, where $t_{i}(r)$ is the 'efficiency function' for the $i$ th task, and $\alpha_{i}$ is a quantity that determines the importance of the $i$ th task for colony fitness. Thus, the quantity $\alpha_{i} t_{i}(r)$ is equivalent to the utility function $\mathcal{U}_{i}$ in our framework. Hasegawa uses the product of individual efficiency functions to arrive at the net efficiency. This means that a small number of unimportant tasks can heavily influence the net efficiency, which is unrealistic. We thus believe that our formulation is more robust to the existence of relatively unimportant tasks.

\subsection{Potential experiments to determine utility and productivity functions}

Determining utility functions and productivity functions for ant colonies is no easy task, and will require elegant experiments, and quite possibly novel experimental techniques altogether. However, if these functions can be determined empirically, our model should provide a framework to predict the future direction of evolution that is likely to be followed by this colony.

Our model also makes one very general prediction, that should be true of all colonies:

We propose that the model can be tested by experimentally generating 'knock out' colonies (the term being borrowed from genetics), by selectively 
eliminating all members of a given caste from a colony of ants, and then eliminating all subsequent larvae that mature into the focal caste. Set up with the appropriate controls, this will allow us to look for 'task redistribution', whereby some ants move away from their task to perform the task that the eliminated caste used to perform. This is a natural prediction of our model (independent of the form of productivity and utility functions). The presence of task redistribution would lend credibility to our model, and the absence of any redistribution, even among morphologically similar castes, would falsify it. Furthermore, if we had access to a large enough number of colonies so as to generate a sizable number of knockout colonies for each caste present in the species, we would be able to directly measure utility functions of these castes by monitoring colony functioning for each class of knockout colony. Specific measures of utility and productivity functions will allow for very specific predictions about quantities such as colony size, caste ratios in a colony, number of castes, etc.

\subsection{Proxies of Utility and Productivity functions}

For a colony of given size $n$, the gross productivity function is $\mathcal{G}=n g$, and the total energy consumed is $\mathcal{E}=n \overline{\mathcal{E}}$. We can attempt to measure utility functions for colonies with unspecialised workers, i.e. colonies where workers are morphologically identical. From equation 3.1 , we know that

$$
\frac{\partial \mathcal{U}_{1}}{\partial \epsilon_{1}}=\frac{\partial \mathcal{U}_{2}}{\partial \epsilon_{2}}=\ldots=\frac{\partial \mathcal{U}_{1}}{\partial \epsilon_{n}}
$$

Now let the per capita utility for task $\mathcal{U}_{i}$ be $\bar{U}_{i}$, therefore,

$$
\mathcal{U}_{i}=n \epsilon_{i} \bar{U}_{i} \Longrightarrow \frac{\partial \mathcal{U}_{i}}{\partial \epsilon_{i}}=n \bar{U}_{i}
$$

But since we know that the fractional marginal utilities of all tasks must be equal, it must mean $n \bar{U}_{i}$ is a constant, which means the per capita utility of each task is independent of the task in question. And since the ants are morphologically identical, the mass of all ants doing a particular task can be used as a proxy for the total utility of that task

$$
\mathcal{U}_{i}=\frac{m_{i}}{M} \bar{U}
$$

where $m_{i}$ is the total mass of all ants engaged in task $i$ and $M$ is the total biomass of the colony. Since the utilities of individual tasks add up to give the gross productivity function, the biomass of the colony can be used as a proxy for measuring the gross productivity function.

Note that the idea that eusocial insect societies are comparable to a superorganism, and thus may be subject to selection at a colony-level, has been proposed before[6][7][8]. The idea that small mutations may be buffered by the rest of the colony has also been proposed before[15]. The idea that caste ratios may be tuned to optimal values is not new either[17][18]. We simply develop a single 
unifying framework that can explain all of these phenomena, and includes all these theories within it. Our model is also consistent with several other empirical observations, and could possibly be used to make precise predicitions about the evolvability of a species, if utility and productivity functions are measured.

\section{Declaration of Competing Interest}

The authors declare that they have no competing interests that may influence the work presented in the paper.

\section{Acknowledgements}

We would like to thank Dr. Sutirth Dey and Dr. Raghavendra Gadagkar for their valuable comments on the manuscript.

\section{Appendix A. Further predictions and empirical observations that are consistent with our model}

\section{Appendix A.1. The total number of castes has an upper bound}

Our model shows that as the number of existing castes in a colony increases, the probability of yet another novel caste evolving decreases.

Let there be $\chi$ castes in a colony, and let $\chi_{i}^{\prime}$ be the number of castes which have the ability to perform a task $\tau_{i}$, and $\chi_{i}<\chi_{i}^{\prime}$ be the number of castes which actually perform the task.

Mutations affect individuals as described in the previous section. Here, we assume the worst possible case, and say that until time $\theta_{-}$(as defined earlier) has passed, the utility of these mutated ants is equal to that of a dead ant. If some fraction $\delta \epsilon_{i}^{\prime}$ of a particular caste that actually perform $\tau_{i}$ is affected by this mutation, we would expect redistribution of workers from the total number of ants from $\chi_{i}^{\prime}$ castes (since the other ants cannot perform $\tau_{i}$ ). From this, we can draw two inferences:

Firstly, since castes are generally specialised in terms of morphology and behavior (and thus the functions they perform), an increased number of castes will likely cause a reduction in the number of castes that can perform a task. Thus it is reasonable to make the following assumption:

Assumption 9. As the number of castes in a colony increases, the number of individuals which can perform a given task, either decreases or stays constant but does not increase, i.e.

$$
\frac{\partial \epsilon_{i}}{\partial \chi} \leq 0 \forall \tau_{i} \in \mathrm{T}
$$


Under this assumption, we can see that the effectiveness of the buffering as described in section 3.2 reduces as the number of castes increases, and thus, if a colony has more pre-existing specialised castes, the detrimental effect on the fitness of the colony during the $\theta_{-}$period is stronger, and the population is more likely to go extinct before the trait can become useful.

Secondly, in the event where certain tasks are essential for sustenance of the colony, a minimum utility may be essential for the survival of the colony, in which case, the population will survive only if the utility of that task exceeds a minimum threshold ${ }^{0} \mathcal{U}_{i}$. Thus the condition to be met after redistribution is:

$$
\mathcal{U}_{i}=m_{i} \bar{v}_{m}+\left(n_{i}-m_{i}\right) \bar{v}_{n m}>{ }^{0} \mathcal{U}_{i}
$$

Where $m_{i}$ is the number of mutants, $n_{i}=\epsilon_{i} . n$ is the total number of ants which can perform $\tau_{i}$, and $\bar{v}_{m}$ and $\bar{v}_{n m}$ are the per capita utilities of the mutants and non mutants respectively. Differentiating with respect to $\epsilon_{i}$, we get:

$$
\frac{\partial \mathcal{U}_{i}}{\partial \epsilon_{i}}=n \bar{v}_{n m}>0
$$

But as a consequence of assumption (9), we see that:

$$
\frac{\partial \epsilon_{i}}{\partial \chi} \leq 0
$$

Therefore from equations (A.1) and (A.2), we see that:

$$
\frac{\partial \mathcal{U}_{i}}{\partial \chi} \leq 0
$$

Equation (A.3) tells us that as the number of castes in a colony increases, $\mathcal{U}_{i}$ decreases, and hence, it becomes harder to keep the utility of these tasks above the minimum utility required for colony maintenance.

\section{Appendix A.2. Variation of number of castes with colony size}

In section 3.3, we illustrated the mechanism through which novel castes can evolve. Integral to this mechanism is the 'buffering capacity' of non-mutated ants, which can prevent the colony from going extinct before the trait becomes beneficial. It is clear that the buffering capacity is higher if the colony includes more non-mutated ants, in the inital stages of evolution. Thus, all other things being equal, we would expect species with larger colony sizes to have a stronger buffering capacity, and thus, it is more probable for a species with a larger colony size to evolve a new caste. Iwasa and Yamaguchi state that ants with larger colonies tend to have more castes [26][27], which is in line with this argument. 


\section{Appendix A.3. Variation in gyne to worker ratio with colony size}

Schmidt et.al. reported that in the Pharoah ant (Monomorium pharaonis), a species that is polygynous and exhibits extensive intranidal mating, the gyne to worker ratio decreases as colony size increases [28].

In a completely intranidal colony, since a single male can fertilize multiple gynes, it would be fair to approximate the scaling of the number of offspring produced by a colony to be linear with respect to the number of gynes. Thus, for a colony of size $n$, if we assume generations are discrete in time, then, at any point $t$ :

$$
n(t+1)=a \cdot n_{g}(t)+b
$$

Where $a$ and $b$ are positive constants, and the generation time is taken to be 1 for simplicity. Thus, an increase in the number of gynes will lead to a linear increase in colony size $n$. Now, for optimal functioning of a colony, we assume that the net productivity $\mathcal{P}$ must be maximized, given $n$, or the per capita net productivity $p$ nust be maximised. We know that:

$$
\begin{aligned}
& \left(\frac{\Delta \mathcal{P}}{\Delta n}\right) \approx p \\
\Longrightarrow & \Delta \mathcal{P} \approx p \cdot \Delta n
\end{aligned}
$$

Thus, a bigger colony will require a proportionally bigger net productivity $\mathcal{P}$ to be able to sustain itself at optimal levels. But because of the law of diminishing marginal utility, we know that we will need a non-linear increase in the number of workers to achieve the same linear increase in net productivity, and thus the number of workers will have to be several times more in the bigger colony to be able to sustain it. Thus, we expect the gyne to worker ratio to decrease as colony size increases, which is what lines up with the observations of Schmidt et.al.

\section{Appendix A.4. Variation of number of castes with latitude}

Wilson [6][18] pointed out that species that live in tropical climates tend to show much higher levels of caste diversity and specialisation than species found in temperate climates. Our model allows for this as well.

In section 3, we showed how our model allows for the evolution of novel castes. We also highlighted that if the mutated trait proves to be useful, selection pressure would lead to those colonies being selected which have that trait. If we consider a utility function $\mathcal{U}(x)$ as a function of the value of a trait $x$, we would expect selection pressure to eventually move the system towards a state with greater $\mathcal{U}(x)$, and thus, natural selection would select the colony which had ants that displayed the phenotype at which $\mathcal{U}$ has a local maximum. This introduces a directionality in the evolution of morphological variations.

However, as we saw earlier, for any given novel (individual-level) trait, there is an initial period when the trait is not useful for the colony, and mutated ants 
thus pose an additional a cost to the colony. However, since the magnitude of benefit is dependent on the utility function, variation in the utility function will lead to variation in the utility of the phenotype. Thus, the directionality that had been induced is lost if the utility function varies, and it is unlikely that the trait reaches the point where it becomes beneficial before being wiped out, in the manner described in section 3. Note that the utility function of any trait is strongly dependent on the environmental conditions, and thus, in temperate climates, which experience greater fluctuations in abiotic factors as seasons change, we would expect fewer castes to be present, and we would expect that those castes which are present are generalists (i.e are not too phenotypically different from the workers) and not specialists, which is in line with Wilson's observation.

\section{Appendix A.5. Multicellularity}

This framework that we propose is a general one, based on basic economic principles, and can be applied to biological systems at various levels. While this document focuses on ant colonies, for the purposes of our model, eusocial insect colonies and primitive multicellular organisms are functionally equivalent. A multicellular organism behaves very similar to a colony: All the cells carry the same genes; Only a few cells reproduce, and the rest serve to maintain optimal functioning of the organism. Thus, we make the bold claim that the same arguments proposed here also serve to explain how differentiation could evolve in multicellular organisms, with the 'castes' in that case refferring to the number of cell types. It has been widely observed that the number of cell types tends to increase as the number of cells increases [26][29]. In the context of our model, this makes sense, since an increase in the number of cells corresponds to a larger buffering effect, and hence a higher likelihood of evolution of novel cell types.

\section{References}

[1] M. J. West-Eberhard, "Developmental plasticity and the origin of species differences," Proceedings of the National Academy of Sciences, vol. 102, no. suppl 1, pp. 6543-6549, 2005.

[2] M. E. Borrello, "The rise, fall and resurrection of group selection," Endeavour, vol. 29, no. 1, pp. 43-47, 2005.

[3] A. Gardner and A. Grafen, "Capturing the superorganism: a formal theory of group adaptation," Journal of evolutionary biology, vol. 22, no. 4, pp. 659-671, 2009.

[4] C. Hou, M. Kaspari, H. B. Vander Zanden, and J. F. Gillooly, "Energetic basis of colonial living in social insects," Proceedings of the National Academy of Sciences, vol. 107, no. 8, pp. 3634-3638, 2010. 
[5] J. F. Gillooly, C. Hou, and M. Kaspari, "Eusocial insects as superorganisms: insights from metabolic theory," Communicative 83 integrative biology, vol. 3, no. 4, pp. 360-362, 2010.

[6] E. O. Wilson, Sociobiology: The New Synthesis. Belknap Press of Harvard University Press, 3rd printing of the 1st edition ed., 1975, 1978.

[7] D. S. Wilson and E. O. Wilson, "Rethinking the theoretical foundation of sociobiology," The Quarterly review of biology, vol. 82, no. 4, pp. 327-348, 2007.

[8] H. K. Reeve and B. Hölldobler, "The emergence of a superorganism through intergroup competition," Proceedings of the National Academy of Sciences, vol. 104, no. 23, pp. 9736-9740, 2007.

[9] S. Powell, "Ecological specialization and the evolution of a specialized caste in Cephalotes ants," Functional Ecology, vol. 22, no. 5, pp. 902-911, 2008.

[10] J. Peat, J. Tucker, and D. Goulson, "Does intraspecific size variation in bumblebees allow colonies to efficiently exploit different flowers?," Ecological Entomology, vol. 30, no. 2, pp. 176-181, 2005.

[11] D. L. Stern and W. A. Foster, "The evolution of soldiers in aphids," Biological Reviews, vol. 71, no. 1, pp. 27-79, 1996.

[12] B. J. Crespi, "Eusociality in australian gall thrips," Nature, vol. 359, no. 6397 , pp. $724-726,1992$.

[13] Y. Cruz, "A sterile defender morph in a polyembryonic hymenopterous parasite," Nature, vol. 294, no. 5840, pp. 446-447, 1981.

[14] R. F. Hechinger, A. C. Wood, and A. M. Kuris, "Social organization in a flatworm: trematode parasites form soldier and reproductive castes," Proceedings of the Royal Society B: Biological Sciences, vol. 278, no. 1706, pp. 656-665, 2011.

[15] M. Molet, D. E. Wheeler, and C. Peeters, "Evolution of novel mosaic castes in ants: Modularity, phenotypic plasticity, and colonial buffering," American Naturalist, vol. 180, no. 3, pp. 328-341, 2012.

[16] S. Londe, T. Monnin, R. Cornette, V. Debat, B. L. Fisher, and M. Molet, "Phenotypic plasticity and modularity allow for the production of novel mosaic phenotypes in ants," Evodevo, vol. 6, no. 1, pp. 1-15, 2015.

[17] G. F. Oster and E. O. Wilson, Caste and ecology in the social insects. Princeton University Press, 1978.

[18] E. O. Wilson, "The ergonomics of caste in the social insects," The American Naturalist, vol. 102, no. 923, pp. 41-66, 1968. 
[19] E. Hasegawa, "The optimal caste ratio in polymorphic ants: Estimation and empirical evidence," American Naturalist, vol. 149, no. 4, pp. 706-722, 1997.

[20] L. Passera, E. Roncin, B. Kaufmann, and L. Kellert, "Increased soldier production in ant colonies exposed to intraspecific competition," Nature, vol. 379, no. February, pp. 630-631, 1996.

[21] J. A. Harvey, L. S. Corley, and M. R. Strand, "Competition induces adaptive shifts in caste ratios of a polyembryonic wasp," Nature, vol. 406, no. 6792 , pp. $183-186,2000$.

[22] T. Kamiya and R. Poulin, "Caste ratios affect the reproductive output of social trematode colonies," Journal of Evolutionary Biology, vol. 26, no. 3, pp. 509-516, 2013.

[23] M. M. Lloyd and R. Poulin, "Reproduction and caste ratios under stress in trematode colonies with a division of labour," Parasitology, vol. 140, no. 7, pp. 825-832, 2013.

[24] M. M. Lloyd and R. Poulin, "Geographic variation in caste ratio of trematode colonies with a division of labour reflect local adaptation," Parasitology Research, vol. 113, no. 7, pp. 2593-2602, 2014.

[25] C. MacLeod, R. Poulin, and C. Lagrue, "Save your host, save yourself? caste-ratio adjustment in a parasite with division of labor and snail host survival following shell damage," Ecology and evolution, vol. 8, no. 3, pp. 1615$1625,2018$.

[26] Y. Iwasa and S. Yamaguchi, "Task allocation in a cooperative society: specialized castes or age-dependent switching among ant workers," Scientific reports, vol. 10, no. 1, pp. 1-9, 2020.

[27] H. Ferguson-Gow, S. Sumner, A. F. Bourke, and K. E. Jones, "Colony size predicts division of labour in attine ants," Proceedings of the Royal Society B: Biological Sciences, vol. 281, no. 1793, p. 20141411, 2014.

[28] A. M. Schmidt, T. A. Linksvayer, J. J. Boomsma, and J. S. Pedersen, "Queen-worker caste ratio depends on colony size in the pharaoh ant (monomorium pharaonis)," Insectes sociaux, vol. 58, no. 2, pp. 139-144, 2011.

[29] J. T. Bonner, The evolution of complexity by means of natural selection. Princeton University Press, 1988. 